\title{
CUERPO EXTRAÑO VEGETAL INTRATESTICULAR
}

\author{
F.M. SÁNCHEZ-MARTÍN*, J. MARTÍ MESTRE*, R. BOSCH PRINCEP**, \\ J. CORBELLA ALONSO****, A. CAMPS PEMÁN***, M. MENDOZA CÁRCAMO*,

\begin{abstract}
*Servicio de Urología. **Servicio de Anatomía Patológica. ***Radiodiagnóstico. Hospital Verge de la Cinta.
\end{abstract} \\ Institut Català de la Salut. Tortosa (Tarragona). ****Ldo. Biología-Botánica. Barcelona.
}

Actas Urol Esp. 28 (1): 65-69, 2004

\section{RESUMEN \\ CUERPO EXTRAÑO VEGETAL INTRATESTICULAR}

Se presenta un caso de cuerpo extraño de origen vegetal ubicado en el parénquima testicular. Se trata de un hallazgo anatomopatológico inesperado en una pieza de orquiectomía realizada con motivo de la atrofia testicular secundaria a una torsión de cordón muy evolucionada. El hallazgo de un material vegetal dentro del testículo es excepcional y, en nuestro conocimiento, no existen otras comunicaciones al respecto. Se discuten los aspectos anatomopatológicos, incluida la reacción granulomatosa presente, así como las cuestiones relacionadas con la atrofia testicular y el mecanismo de entrada del cuerpo extraño. La manipulación del escroto por parte de curanderos durante la fase de atrofia testicular explica la presencia de este material vegetal intratesticular.

PALABRAS CLAVE: Cuerpo extraño. Testículo. Atrofia testicular. Paramedicina.

\section{ABSTRACT}

\section{VEGETABLE FOREIGN BODY IN THE TESTIS}

A vegetable foreign body located inside the testicular parenchyma is presented. It's about an unexpected pathological finding in an orchidectomy sample, performed on account of testicular atrophy secondary to very advanced funicular twist. The finding of a vegetal material inside the testis is outstanding. In our knowledge we don't know about another similar case. Pathologic aspects, including granulomatous reaction, regarding arguments of testicular atrophy and the foreign body irruption mechanism are discussed. The handling of the scrotum by paramedical people could justify his presence.

KEY WORDS: Foreign body. Testicle. Testicular atrophy. Paramedicine.

L os cuerpos extraños alojados en las paredes de la bolsa escrotal son reportados con cierta frecuencia en la literatura médica ${ }^{1,3}$, mientras que los estrictamente intratesticulares son menos frecuentes. El hallazgo de un material vegetal intratesticular es excepcional y, en nuestro conocimiento, no existe otra comunicación al respecto. La movilidad del testículo explica la dificultad para que procesos externos alcancen su parénquima. El probable mecanismo de introducción del vegetal atribuible a personajes dedicados a la curandería aporta un elemento más de interés a este caso.

\section{CASO CLÍNICO}

Paciente de 15 años que consultó en urgencias por escroto agudo derecho de más de 48 horas de evolución. Se apreció un escroto muy empastado y 
tumefacto, con pérdida de los pliegues cutáneos, en el que tanto el testículo como el epidídimo eran difíciles de diferenciar y resultaban muy dolorosos. No había fiebre ni molestias miccionales. En la analítica destacaba leucocitosis de 14,9 x $10^{3} / \mu$ l con $83 \%$ de neutrófilos, y en orina $8-10$ leucocitos por campo. La ecografía escrotal mostró una marcada disminución en la ecogenicidad del parénquima testicular derecho respecto del izquierdo y un importante engrosamiento de la cabeza del epidídimo. No se disponía de dopplercolor.

Se decidió conducta conservadora con antiinflamatorios y antibióticos ante la larga evolución del cuadro. En semanas sucesivas se observaron intensos cambios inflamatorios escrotales, con ligero exudado cutáneo. Los marcadores tumorales fueron negativos. El cuadro inflamatorio fue cediendo y en la ecografía realizada a los 5 meses apareció un testículo derecho atrófico (2 cm Ø) difícil de diferenciar del epidídimo (Fig. 1). A la palpación el teste era pequeño y duro, por lo que se propuso orquiectomía y colocación de una prótesis testicular.

La intervención tuvo lugar 6 meses después del episodio agudo y se practicó bajo anestesia general. Por vía inguinal se halló tan sólo un vestigio epididimotesticular derecho sin ningún otro dato destacable. La orquiectomía se realizó mediante ligadura en bloque del cordón esper-

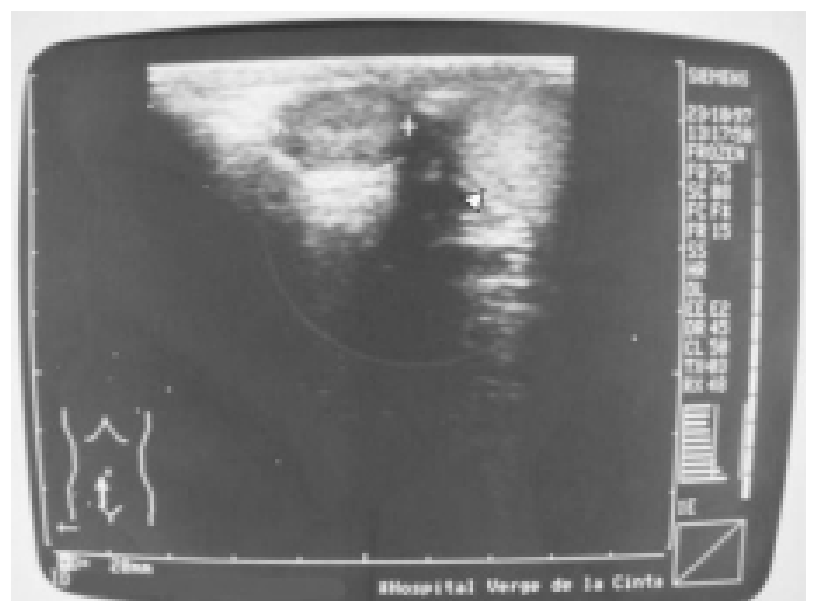

FIGURA 1. Testiculo derecho (flecha) en proceso de atrofia completa, muy disminuido de tamaño respecto del testiculo izquierdo normal (punta de flecha). En esta fase no se observan imágenes extrañas dentro del parénquima testicular. mático con hilo de ácido poliglicólico y la pieza se introdujo de forma inmediata en formol. Se colocó prótesis testicular de silicona vulcanizada que se fijó con un punto de seda a la darthos. La pared se cerró con suturas reabsorbibles de poliglicólico en dos planos y la piel con subcuticular de polipropileno. También se practicó fijación testicular izquierda con poliglicólico vía transescrotal. No se dejaron drenajes. El post-operatorio transcurrió sin incidencias con alta a las 24 horas.

El estudio anatomopatológico informó: pieza quirúrgica de 40 × $20 \mathrm{~mm}$, de superficie lisa y brillante y de coloración blanquecina que presenta al corte un testículo atrófico de $15 \mathrm{~mm}$ de diámetro máximo, de consistencia aumentada y coloración parda. Entre el epidídimo y el parénquima testicular se observa una zona de 5 x $5 \mathrm{~mm}$ de consistencia mayor y coloración marrón. El estudio microscópico revela un parénquima testicular con zonas necróticas rodeadas por parénquima testicular sustituido por tejido fibroso sin presencia de celularidad intersticial ni tubular. No se observan elementos infecciosos ni infiltrados inflamatorios significativos. Destaca la presencia de material extraño en forma de reticulado que se tiñe con la tinción de PAS, es resistente a la digestión con diastasa y presenta una leve birrefringencia, lo que corresponde a fibras vegetales (Fig. 2). Este material extraño se halla en contacto con el parénquima testicular cercano al origen del epidídimo, y está rodeado por una reacción granulomatosa gigantocelular a cuerpo extraño, no observándose zonas de necrosis caseosa, necrosis grasa (células xánticas), restos de estructuras epiteliales, ni restos pilosos o queratina. Las tinciones de Zielh-Neelsen para micobacterias resultan negativas. Cortes longitudinales del cuerpo extraño demuestran una formación compacta, laminada y de fractura periférica astillosa (Fig. 3).

En la visita sucesiva se comentó con la madre del chico la ausencia de procesos malignos en la pieza y mediante preguntas se descartaron traumatismos accidentales tanto previos como posteriores al escroto agudo. Acto seguido se preguntó si quizás habían acudido a alguna persona relacionada con la paramedicina o el curanderismo. La madre respondió de forma afirmativa. Habían 


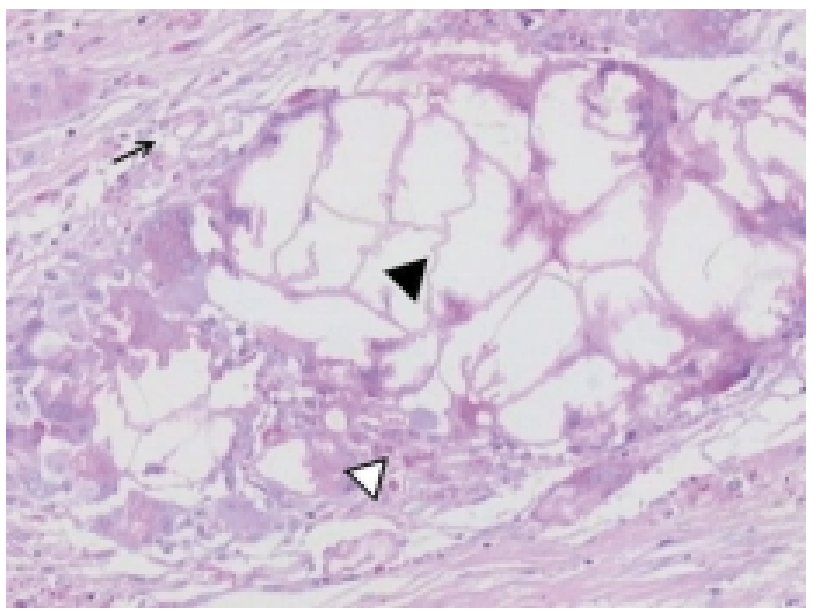

FIGURA 2. Corte transverso del material extraño en el que se aprecia un cúmulo de numerosos gránulos y el trabeculado PAS positivo (punta flecha negra) que recuerda a la celulosa, en contacto con el parénquima testicular (punta flecha blanca). Rodea el material una importante reacción granulomatosa a cuerpo extraño (flecha fina).

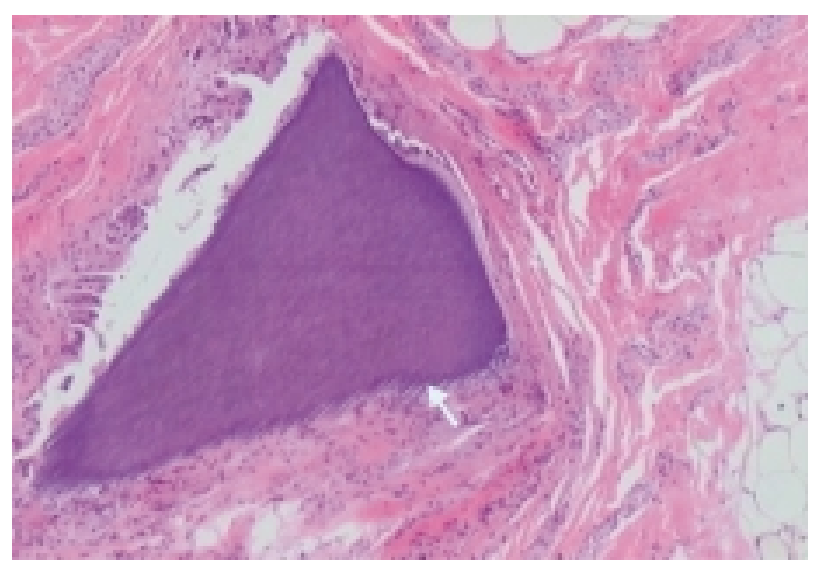

FIGURA 3. Corte longitudinal del material extraño intratesticular donde se aprecia su forma cuneiforme y la densa disposición de sus fibras (flecha). En este corte no es tan evidente la reacción granulomatosa.

acudido varias veces a un "sanador" de la comarca, quien había aplicado diversas maniobras al escroto durante la etapa inflamatorio-degenerativa. Algunas de las manipulaciones habían provocado intenso dolor pero no tenían noción exacta de en qué consistieron. Recurrieron a estos remedios al ver la tórpida evolución del escroto en las semanas que siguieron al episodio agudo. No fue posible obtener información más precisa. Han transcurrido 5 años desde la intervención y el paciente se halla asintomático, aunque se ha detectado oligoastenozoospermia.

\section{DISCUSIÓN}

El presente caso plantea tres líneas de discusión: la presencia de cuerpos extraños intratesticulares, la etiología de la atrofia testicular y el papel que ha podido jugar la paramedicina.

Los cuerpos extraños intratesticulares son muy inusuales. Algunos hallazgos intraescrotales son denominados intratesticulares sin serlo realmente, como en el trabajo clásico de Staff y Lomax $^{1}$. Pese a que la movilidad testicular ejerce una función protectora frente a los traumatismos contusos, la glándula puede quedar comprometida si el mecanismo es penetrante, ya que la fina piel del escroto y la laxitud de las cubiertas ofrecen escasa resistencia.

Los materiales extraños más clásicos en contacto con el parénquima testicular son los metálicos, tanto por accidente laboral (martillos, neumáticos) como derivados de conflictos bélicos ${ }^{2}$ (metralla), siendo unos y otros poco frecuentes. Más habitual es que se comuniquen materiales alojados fuera de la glándula, implicando sólo a las cubiertas del escroto $^{3}$, incluida la túnica vaginalis ${ }^{4}$. Son casos de granulomas a cuerpo extraño secundarios a inyección de fluidos de alta viscosidad tanto en pene como en escroto, que se realizan con intención de aumentar el tamaño de los órganos genitales. Los implantes se colocan directamente en el escroto o migran a él desde el pene o incluso las caderas, como en el caso de transexuales que moldean su silueta ${ }^{5}$. Se trata casi siempre de silicona o parafina inyectadas ${ }^{6}$, aunque también son inoculados aceites sin ningún tipo de control sanitario que provocan importantes reacciones locales ${ }^{7}$. La anatomía patológica en el caso de los aceites muestra un granuloma en Y con importante infiltración de eosinófilos, y al microscopio óptico no se observan rastros del aceite aunque sí histiocitos y células gigantes con vacuolas grasas intracitoplasmáticas (lipogranuloma esclerosante) ${ }^{8}$. El talco de los guantes depositado inadvertidamente en el lecho quirúrgico de cirugía escroto-inguinal puede provocar granulomas a largo plazo $^{9}$ en las túnicas, incluso con aspecto de una masa. Su característica es la polarización del talco en "cruz de malta". El tricobezoar es otra posibilidad de cuerpo extraño intraescrotal, con presencia de restos pilosos y la reacción a 
cuerpo extraño a partir de la queratina del propio paciente. También están descritos materiales extraños libres en el espacio vaginal coincidiendo con un teratoma intraescrotal ${ }^{10}$.

Sólo existe una referencia en la literatura a cuerpo extraño vegetal, aunque no intratesticular sino adherido a la túnica vaginalis ${ }^{11}$. Se trata de un paciente que, meses después de una intervención por hernia inguinal incarcerada y perforación yeyunal, presentó inflamación escrotal. Era la reacción a cuerpo extraño debida a restos de fibras vegetales en las cubiertas escrotales. Los hallazgos histológicos son muy similares a los de nuestro caso (gránulos de celulosa y reacción a cuerpo extraño).

El diagnóstico de materia vegetal se basa en la imagen histológica repetida en varios cortes de la pieza de orquiectomía, donde el voluminoso cuerpo protuye en el parénquima testicular fibrosado (Fig. 3). La importante cantidad de celulosa representada por el material PAS positivo, diastasa resistente y con una morfología reticular particular hace evidente su origen vegetal ya que no existen materiales animales (catgut, seda) o minerales (talco, aleaciones, silicona) que presenten dicha morfología. Tampoco se trata de material quirúrgico de origen vegetal (algodón). La reacción a cuerpo extraño sólo es posible si se produce antes de la cirugía, lo que descarta la contaminación peroperatoria. Tampoco se usaron durante el acto quirúrgico materiales de sutura de origen vegetal (lino) o animal (catgut), y la seda se empleó sólo para fijar la prótesis una vez extirpada la pieza. La reacción granulomatosa presente en nuestro caso es la típica del cuerpo extraño. Se trata de histiocitos de citoplasma esmerilado con núcleos distribuidos uniformemente en la periferia. Es diferente de otros granulomas como los observados en testículos con secuelas isquémicas o de tuberculosis donde los núcleos se disponen en herradura ${ }^{12}$. Por su parte la xantogranulomatosis (necrosis grasa) presenta histiocitos con citoplasma claro y espumoso y núcleos agrupados en el centro. Con el material disponible no fue posible establecer la taxonomía del vegetal.

La secuencia más probable en nuestro caso es la de torsión testicular evolucionada, isquemia y necrosis, introducción de cuerpo extraño (paramedicina), reacción a cuerpo extraño y atrofia definitiva. La ausencia de hematoma en todo el proceso da pie a afirmar que el testículo carecía ya de vascularización cuando fue puncionado, si bien está descrita la ruptura puntual de la albugínea sin hemorragia. Nuestro paciente negó haber padecido cualquier trauma previo al cuadro de dolor escrotal y tampoco la ecografía inicial detectó hallazgos representativos, y esta prueba es muy sensible a la hora de demostrar cuerpos extraños o rotura testicular ${ }^{13}$. El grado de participación del material extraño en la atrofia puede ser discutido ya que los traumatismos penetrantes del teste han sido referidos como causa de atrofia testicular ${ }^{14}$. Superado ya el mito de que la retracción del testículo era inevitable si la albugínea era perforada, el trabajo de Cross et al. demuestra cambios atróficos en 5 de 10 traumas escrotales ${ }^{15}$, aunque para Bautista et al. ningún testículo se atrofió en su serie de 41 traumatismos ${ }^{16}$.

La posibilidad de que el cuerpo extraño vegetal provenga de una manipulación escrotal en el ámbito de la paramedicina nos parece la hipótesis más factible, ya que la familia y el niño reconocieron la actuación de un curandero, con manipulación muy dolorosa del escroto una vez éste se hallaba en pleno proceso necrótico. Es cada vez más frecuente que los pacientes acudan a remedios al margen de la medicina convencional, con objeto de solucionar sus problemas de salud. En nuestro país un $45 \%$ de los enfermos de asistencia primaria reconocen haber recurrido a la medicina alternativa, con un $37 \%$ de visitas a curanderos/sanadores ${ }^{17}$. Pese a que sólo el $18 \%$ reconoce haber mejorado, se observa que estos recursos son cada vez más usados. La medicina alternativa es un fenómeno emergente cuyo gasto alcanzó en USA los 12,2 billones de dólares anuales (pagados del propio bolsillo de los pacientes) ${ }^{18}$. En urología también puede apreciarse el impacto de la paramedicina en la práctica urológica convencional, donde un $13 \%$ de los pacientes han recibido terapias alternativas ${ }^{19}$.

Ante casos como el nuestro es fácil caer en el error de maljuzgar la iniciativa del paciente o su familia de acudir a soluciones paramédicas. Las cuestiones culturales subyacentes en la población enferma y las limitaciones de los sistemas sanitarios hacen comprensible que se recurra a 
soluciones diferentes a la medicina tradicional. Como señalan Turabiánaa y Pérez-Francob, existen muchos más actores que los propiamente médicos implicados en la solución de problemas de salud en la comunidad ${ }^{20}$. Las soluciones no son siempre formales ya que el paciente busca ayuda acudiendo cada vez con más asiduidad a foros que poco tienen que ver con el canon científico. Hay que recordar que la enfermedad individual depende de contextos, lo que a su vez hace reaccionar dichos contextos. No todo queda limitado al ámbito de la medicina. La presencia de personajes diferentes al médico en la atención a la salud debe ser comprendida en sus justos términos. Según Luna y Ruiz-Moral, los pacientes no sólo sufren su proceso de una forma pasiva, sino que le otorgan un significado vital donde la dolencia es interpretada en términos existenciales, interrelacionando con el entorno y no como algo aislado. El enfermo participa de muchas y diferentes influencias, algo que no puede ser ignorado por el médico ${ }^{21}$.

Agradecimientos: Al Dr. Ferrán Algaba por sus precisiones sobre las imágenes anatomopatológicas.

\section{REFERENCIAS}

1. STAFF WG, LOMAS AJ.: Foreign bodies in the testis. Brit J Surg 1968; 55 (4): 255-256.

2. LEARCH TJ, HANSCH LP, RALLS PW.: Sonography in patients with gunshot wounds of the scrotum: imaging findings and their value. Am $J$ Roentgenol 1995 oct; 165 (4): 879-883.

3. WASSERMANN RJ, GREENWALD DP.: Debilitating silicone granuloma of the penis and scrotum. Plast Surg 1995; 35 (5): 505-509.

4. HEALEY GB, MCDONALD DF.: Talc granuloma presenting as a testicular mass. J Urol 1977; 118: 122.

5. FARIÑA LA, PALACIO V, SALLES M, FERNÁNDEZVILLANUEVA D, VIDAL B, MENÉNDEZ P.: Granuloma escrotal por migración desde la cadera en 2 varones transexuales. Arch Esp Urol 1977 janfeb; 50 (1): 51-53.

6. PODLUZHNYI GA, TIGOV AD, BRAGANETS AM, IAKIMENKO VA.: The clinical picture, classification and surgical treatment of paraffinomas of the external genitalia (abstract). Urol Nefrol (Mosk) 1991; 4: 69-73.

7. BEHAR TA, ANDERSON EE, BARWICK WJ, MOHLER JL.: Sclerosing lipogranulomatosis: a case report of scrotal injection of automobile. Plast Reconstr Surg 1993 feb; 91 (2): 352-361.
8. MATSUDA T, SHICHIRI Y, HIDA S, OKADA Y, TAKEUCHI H, NAKASHIMA Y, YOSHIDA O.: Eosinophilic sclerosing lipogranuloma of the male genitalia not caused by exogenous lipids. J Urol 1988; 140 (5): 1021-1024.

9. MILLER RA, KIVIAT MD, GRAVES J.: Glove-starch granuloma in congenital hydrocele. Urology 1974 may; 3 (5): 610-611.

10. NINI W, NINI N.: Intrascrotal teratoma forming a free foreign body in the tunica vaginalis. A propos of a case. Chirurgie 1988; 114: 268-269.

11. MARTÍNEZ ALARCÓN M, MIRANDA C, ESTEFANO C.: Inflamación intraescrotal crónica secundaria a cuerpo extraño en la túnica vaginalis. Arch Esp Urol 1993 jun; 46 (5): 429-431.

12. NISTAL M, MATE A, PANIAGUA R.: Granulomatous epididimal lesions of possible ischemic origin. Am J Surg Pathol 1997; 21 (8): 951-956.

13. LEARCH TJ, HANSCH LP, RALLS PW.: Sonography in patients with gunshot wounds of the scrotum: imaging findings and their value. AJR Am J Roentgenol 1995 oct; 165 (4): 879-883.

14. MACDERMOTT JP, GRAY BK.: Bilateral testicular atrophy following blunt trauma. Br J Urol $1989 \mathrm{feb}$; 63 (2): 215-216.

15. CROSS JJ, BERMAN LH, ELLIOTT PG, IRVING S.: Scrotal trauma: a cause of testicular atrophy. Clin Radiol 1998 may; 54 (5): 317-320.

16. BAUTISTA A, ALVEZ GONZÁLEZ F, IGLESIAS JL, VILLANUEVA A, GARCÍA GARCÍA E, LEIS R, VARELA R.: Traumatismos escrotales en la infancia. An Esp Pediatr 1992 dec; 37 (6): 477-480.

17. CARRETERO JL, RUIZ A, YAGÜE E, PÉREZ MARTÍN RN.: Medicina alternativa frente a medicina científica en un área básica de salud. ¿Un fenómeno emergente?. Med Clin 2001; 117: 439-441.

18. EISENBERG DM, DAVIS RB, ETTNER SL, APPEL S, WILKEY S, VAN ROMPAY M, KESSLER RC.: Trends in alternative medicine use in the United States, 1990-1997: results of a follow-up national survey. JAMA 1998 nov 11; 280 (18): 1569-1575.

19. STRATEV S, PANAIOTOV P.: The problem of paramedicine and the therapy of urologic patients (abstract). Khirurgiia (Sofia) 1993; 46 (4): 54-57.

20. TURABIÁNAA JL, PÉREZ-FRANCO B.: ¿Cuál es el sentido de la educación para la salud y las actividades "comunitarias" en atención primaria?. Atención Primaria 1998; 22: 662-666.

21. LUNA L, RUIZ MORAL R.: Una clarificación conceptual de las nuevas propuestas en medicina de familia. Atención Primaria 2000; 26: 98-100.

Dr. F.M. Sánchez Martín

Urb. Tres Palmeres, 6 - Sector J

43530 Alcanar Platja (Tarragona)

(Trabajo recibido el 26 mayo de 2003) 